\title{
Enumeration of I-graphs: Burnside does it again
}

\author{
Marko Petkovšek \\ University of Ljubljana, Faculty of Mathematics and Physics \\ Jadranska 19, SI-1000 Ljubljana, Slovenia \\ Helena Zakrajšek \\ University of Ljubljana, Faculty of Mechanical Engineering \\ Aškerčeva 6, SI-1000 Ljubljana, Slovenia
}

Received 21 July 2009, accepted 12 November 2009, published online 17 December 2009

\begin{abstract}
We give explicit and efficiently computable formulas for the number of isomorphism classes of I-graphs, connected I-graphs, bipartite connected I-graphs, generalized Petersen graphs, and bipartite generalized Petersen graphs. The tool that we use is the well-known Cauchy-Frobenius-Burnside lemma.
\end{abstract}

Keywords: I-graphs, generalized Petersen graphs, Cauchy-Frobenius-Burnside lemma, arithmetical functions.

Math. Subj. Class.: 05A15, 05C30

\section{Introduction}

Recently the class of I-graphs, introduced in the Foster Census [2] as a further development of the generalized Petersen graphs, has received considerable attention. One reason for this is that bipartite I-graphs give rise to some highly symmetric configurations of points and lines [1]. In the same paper, Boben, Pisanski and Žitnik characterized the automorphism groups of those I-graphs which are not generalized Petersen graphs, so that together with the earlier results of Frucht, Graver and Watkins [3], the characterization of the automorphism groups of I-graphs is now complete. Finally, Horvat, Pisanski and Žitnik have recently shown that every I-graph has a nondegenerate unit-distance representation in the Euclidean plane [4]. This answers the question of whether every generalized Petersen

E-mail addresses: marko.petkovsek@fmf.uni-lj.si (Marko Petkovšek), helena.zakrajsek@fs.uni-lj.si (Helena Zakrajšek) 
graph can be drawn in the plane in such a way that all edges are represented by straight-line segments of equal length.

As witnessed by the recent inclusion of the corresponding counting sequences in [10], there has also been interest in the enumeration of non-isomorphic I-graphs and various of their subclasses, such as connected I-graphs, generalized Petersen graphs, etc. However, explicit formulas for the $n$-th term of these sequences seem to be unknown, with the sole exception of the formula for the number of non-isomorphic generalized Petersen graphs $G(n, k)$ on $2 n$ vertices with $\operatorname{gcd}(n, k)=1$, given quite recently by Steimle and Staton [12, Thm. 11].

At a seminar meeting in Ljubljana in January 2009, T. Pisanski asked for a formula enumerating non-isomorphic I-graphs on $2 n$ vertices. We give such a formula below in Section 2 , as well as analogous formulas enumerating non-isomorphic connected I-graphs, bipartite connected I-graphs, generalized Petersen graphs, and bipartite generalized Petersen graphs on $2 n$ vertices. These formulas are in closed form, and can be used for efficient computation of the number of isomorphism classes, provided that the prime factorization of $n$ is known.

To enumerate isomorphism classes we use the Cauchy-Frobenius lemma, also known as Burnside's lemma. Although very well known, this lemma is seldom applied directly, but rather indirectly via the Redfield-Pólya enumeration theorem whose proof relies on it. Recently, though, it has been used successfully on its own in several cases (cf. [9, 6, 7]).

For $n \in \mathbb{N}$ write $\mathbb{Z}_{n}=\{0,1, \ldots, n-1\}$ and $\mathbb{Z}_{n}^{\prime}=\mathbb{Z}_{n} \backslash\{0, n / 2\}$. Let $n \in \mathbb{N}, n \geq 3$, and $j, k \in \mathbb{Z}_{n}^{\prime}$. The $I$-graph $I(n, j, k)$ is the graph $G=(V, E)$ where

$$
\begin{aligned}
& V=\mathbb{Z}_{n} \times \mathbb{Z}_{2}, \\
& E=\bigcup_{i=0}^{n-1}\{\{(i, 0),(i, 1)\},\{(i, 0),(i+j, 0)\},\{(i, 1),(i+k, 1)\}\},
\end{aligned}
$$

and addition is performed modulo $n$. Well-known special cases include the $n$-prism $Y_{n}=$ $I(n, 1,1)$, the Petersen graph $I(5,1,2)$, and the generalized Petersen graph $G(n, k)=$ $I(n, 1, k)$, introduced by Watkins in [13].

The I-graph $I(n, j, k)$ is a cubic graph on $2 n$ vertices. In [1], several graph-theoretic properties of $I(n, j, k)$ such as connectedness, girth, being bipartite or being vertex-symmetric, are characterized in terms of number-theoretic properties of parameters $n, j, k$. An algorithm for deciding which sets of parameter values give rise to isomorphic I-graphs is also given there. In [5], the following result (crucial for our enumeration) is proved:

Theorem 1.1. $I(n, j, k)$ and $I\left(n, j^{\prime}, k^{\prime}\right)$ are isomorphic if and only if there exists an integer $a$, relatively prime to $n$, such that either $\left\{j^{\prime}, k^{\prime}\right\}=\{a j \bmod n, a k \bmod n\}$ or $\left\{j^{\prime}, k^{\prime}\right\}=$ $\{a j \bmod n,-a k \bmod n\}$.

We also rely on the following results from [1]:

Theorem 1.2. The graph $I(n, j, k)$ is connected if and only if $\operatorname{gcd}(n, j, k)=1$.

Theorem 1.3. A connected graph $I(n, j, k)$ is bipartite if and only if $n$ is even and $j$ and $k$ are odd.

In the rest of the paper, we use the following notation (for $n \in \mathbb{N}$ ): 


$$
\begin{aligned}
& I(n)=\text { the number of isomorphism classes of I-graphs } I(n, j, k) \\
& \text { (sequence A153846 in [10]) } \\
& I_{c}(n)=\text { the number of isomorphism classes of connected I-graphs } \\
& I(n, j, k) \text { (sequence A153847 in [10]) } \\
& I_{b c}(n)=\text { the number of isomorphism classes of bipartite connected } \\
& \text { I-graphs } I(n, j, k) \\
& P(n)=\text { the number of isomorphism classes of generalized Petersen } \\
& \text { graphs } G(n, k)=I(n, 1, k) \text { (sequence A077105 in [10]) } \\
& P_{b}(n)=\text { the number of isomorphism classes of bipartite generalized } \\
& \text { Petersen graphs } G(n, k)=I(n, 1, k) \text { (sequence A107452 in [10]) } \\
& P_{r}(n)=\text { the number of isomorphism classes of generalized Petersen } \\
& \text { graphs } G(n, k)=I(n, 1, k) \text { with } \operatorname{gcd}(n, k)=1 \\
& \mathbb{Z}_{n}=\{0,1, \ldots, n-1\} \quad \text { (the ring of integers modulo } n \text { ) } \\
& \mathbb{Z}_{n}^{*}=\left\{a \in \mathbb{Z}_{n} ; \operatorname{gcd}(a, n)=1\right\} \text { (the group of units of } \mathbb{Z}_{n} \text { ) } \\
& \mathbb{Z}_{n}^{\prime}=\mathbb{Z}_{n} \backslash\{0, n / 2\} \quad \text { (the set of legal values for } j, k \text { in } I(n, j, k) \text { ) }
\end{aligned}
$$

\begin{tabular}{|c|c|c|}
\hline notation & OEIS id & comments \\
\hline$\mu(n)$ & A008683 & Moebius function \\
\hline$\tau(n)$ & A000005 & the number of divisors of $n$ \\
\hline$\varphi(n)$ & A000010 & $\begin{array}{l}\text { Euler's totient function, } \\
\qquad \varphi(n)=\left|\left\{j \in \mathbb{Z}_{n} ; \operatorname{gcd}(n, j)=1\right\}\right|=\left|\mathbb{Z}_{n}^{*}\right|\end{array}$ \\
\hline$J_{2}(n)$ & A007434 & $\begin{array}{l}\text { the second Jordan's totient function, } \\
\qquad J_{2}(n)=\left|\left\{(j, k) \in \mathbb{Z}_{n} \times \mathbb{Z}_{n} ; \operatorname{gcd}(n, j, k)=1\right\}\right|\end{array}$ \\
\hline$\omega(n)$ & A001221 & the number of distinct prime factors of $n$ \\
\hline$r(n)$ & A060594 & $\begin{array}{l}\text { the number of square roots of } 1 \text { modulo } n \\
\qquad r(n)=\left|\left\{a \in \mathbb{Z}_{n} ; a^{2} \equiv 1(\bmod n)\right\}\right|\end{array}$ \\
\hline$s(n)$ & A000089 & $\begin{array}{l}\text { the number of square roots of }-1 \text { modulo } n \\
\qquad s(n)=\left|\left\{a \in \mathbb{Z}_{n} ; a^{2} \equiv-1(\bmod n)\right\}\right|\end{array}$ \\
\hline
\end{tabular}

For $k \in \mathbb{Z}$, we write $k \bmod n$ to denote the unique $r \in \mathbb{Z}_{n}$ such that $k \equiv r(\bmod n)$. In particular, if $n$ is even, then

$$
(n / 2) \bmod 2=\left\{\begin{array}{lll}
0, & n \equiv 0 \quad(\bmod 4) \\
1, & n \equiv 2 \quad(\bmod 4)
\end{array}\right.
$$

Table 1 lists the arithmetical functions that appear in the rest of the paper. The column "OEIS id" in Table 1 gives the corresponding identifier from [10].

Table 1: Some arithmetical functions.

With the exception of $\omega(n)$ which is additive, all other functions in Table 1 are multiplicative. If $p$ is a prime and $k \geq 1$, we have

$$
J_{2}\left(p^{k}\right)=p^{2 k}-p^{2 k-2}=\sum_{d \mid p^{k}} \mu\left(\frac{p^{k}}{d}\right) d^{2},
$$




$$
\begin{aligned}
& r\left(p^{k}\right)= \begin{cases}1, & p=2 \text { and } k=1, \\
2, & p \text { odd } \text { or }(p=2 \text { and } k=2), \\
4, & p=2 \text { and } k \geq 3,\end{cases} \\
& s\left(p^{k}\right)= \begin{cases}0, & p \equiv 3(\bmod 4) \text { or }(p=2 \text { and } k \geq 2), \\
1, & p=2 \text { and } k=1, \\
2, & p \equiv 1(\bmod 4),\end{cases}
\end{aligned}
$$

hence

$$
\begin{gathered}
J_{2}(n)=n^{2} \prod_{\substack{p \mid n \\
p \text { prime }}}\left(1-\frac{1}{p^{2}}\right)=\sum_{d \mid n} \mu\left(\frac{n}{d}\right) d^{2}, \\
r(n)= \begin{cases}2^{\omega(n)}, & n \equiv 1(\bmod 2) \text { or } n \equiv 4(\bmod 8), \\
2^{\omega(n)-1}, & n \equiv 2(\bmod 4), \\
2^{\omega(n)+1}, & n \equiv 0(\bmod 8),\end{cases} \\
s(n)= \begin{cases}0, & 4 \mid n \text { or } \exists p \operatorname{prime}:(p \mid n \text { and } p \equiv 3(\bmod 4)), \\
2^{\psi(n)}, & \text { otherwise, }\end{cases}
\end{gathered}
$$

where $\psi(n)=\mid\{p \mid n ; p$ prime, $p \equiv 1(\bmod 4)\} \mid$.

The following formula (which can also be proved by our methods) is given in [12, Thm. 11]:

Theorem 1.4. The number $P_{r}(n)$ of isomorphism classes of generalized Petersen graphs $G(n, k)$ on $2 n$ vertices with $\operatorname{gcd}(n, k)=1$ is given by

$$
P_{r}(n)=\frac{1}{4}(\varphi(n)+r(n)+s(n))
$$

In Section 2 we list our formulas for $I(n), I_{c}(n), I_{b c}(n), P(n), P_{b}(n)$ which seem to be new, and tabulate their values (as well as those of $P_{r}(n)$ ) for some small values of $n$. In Section 3 we explain our proof techniques and give the proofs.

\section{The main results}

Theorem 2.1. Let $n=p_{1}^{k_{1}} p_{2}^{k_{2}} \cdots p_{\omega(n)}^{k_{\omega(n)}}$ be the prime factorization of $n$. Then the number of isomorphism classes of I-graphs on $2 n$ vertices is given by

$$
I(n)=\frac{1}{4} \sum_{i=1}^{4} \prod_{j=1}^{\omega(n)} g_{i}\left(p_{j}^{k_{j}}\right)-\left\{\begin{array}{cc}
2 \tau(n)-1, & n \text { even } \\
\tau(n), & n \text { odd }
\end{array}\right.
$$


where

$$
\begin{aligned}
& g_{1}\left(p^{k}\right)=\frac{(p+1) p^{k}-2}{p-1}, \\
& g_{2}\left(p^{k}\right)= \begin{cases}4 k, & p=2, \\
2 k+1, & p>2,\end{cases} \\
& g_{3}\left(p^{k}\right)= \begin{cases}2, & p=2 \text { and } k=1 \\
4(k-1), & p=2 \text { and } k \geq 2, \\
2 k+1, & p>2\end{cases} \\
& g_{4}\left(p^{k}\right)= \begin{cases}2, & p=2, \\
2 k+1, & p \equiv 1 \\
1, & p \equiv 3 \quad(\bmod 4),\end{cases}
\end{aligned}
$$

Theorem 2.2. The number $P(n)$ of isomorphism classes of generalized Petersen graphs on $2 n$ vertices is given by

$$
P(n)=\frac{1}{4}(2 n-\varphi(n)-2 \operatorname{gcd}(n, 2)+r(n)+s(n)) .
$$

Theorem 2.3. The number of isomorphism classes of connected I-graphs on $2 n$ vertices is given by

$$
I_{c}(n)=\frac{1}{4}\left(\frac{J_{2}(n)}{\varphi(n)}+r(n)+s(n)+t(n)\right)- \begin{cases}1, & n \text { odd } \\ 2, & n \equiv 0(\bmod 4) \\ 3, & n \equiv 2(\bmod 4)\end{cases}
$$

where

$$
t(n)= \begin{cases}2^{\omega(n)}+2^{\omega(n / 2)}, & n \text { even } \\ 2^{\omega(n)}, & n \text { odd }\end{cases}
$$

Theorem 2.4. For $n$ even, let $\chi(n)=(n / 2) \bmod 2$. The number of isomorphism classes of bipartite generalized Petersen graphs on $2 n$ vertices is given by

$$
P_{b}(n)= \begin{cases}\frac{1}{4}(n-\varphi(n)-2 \chi(n)+r(n)+s(n)), & n \text { even } \\ 0, & n \text { odd }\end{cases}
$$

Theorem 2.5. For $n$ even, let $\chi(n)=(n / 2) \bmod 2$. The number of isomorphism classes of bipartite connected I-graphs on $2 n$ vertices is given by

$$
I_{b c}(n)= \begin{cases}\frac{1}{4}\left(\frac{J_{2}(n)}{3 \varphi(n)}+\chi(n) 2^{\omega(n / 2)}+r(n)+s(n)\right)-\chi(n), & n \text { even } \\ 0, & n \text { odd } .\end{cases}
$$

Corollary 2.6. Let $p$ be an odd prime. Then

$$
I(p)=I_{c}(p)=P(p)=P_{r}(p)=\left\lceil\frac{p}{4}\right\rceil .
$$




\begin{tabular}{|c|c|c|c|c|c|c|c|c|c|c|c|c|c|c|c|c|c|c|}
\hline$n$ & 3 & 5 & 6 & 7 & 8 & & 10 & 11 & 12 & 13 & 14 & 15 & 1 & $1^{\prime}$ & 18 & 19 & 20 & 21 \\
\hline$I(n)$ & 1 & 2 & 3 & 2 & 4 & & 6 & 3 & 11 & 4 & 7 & 10 & 1 & 5 & 14 & 5 & 17 & 12 \\
\hline$I_{c}(n)$ & 1 & 2 & 2 & 2 & 3 & & 4 & 3 & 7 & 4 & 5 & 7 & c & 5 & 8 & 5 & 10 & 9 \\
\hline$P(n)$ & 1 & 2 & 2 & 2 & 3 & & 4 & 3 & 5 & 4 & 5 & 6 & 6 & 5 & 7 & 5 & 8 & 8 \\
\hline$P_{r}(n)$ & 1 & 2 & 1 & 2 & 2 & & 2 & 3 & 2 & 4 & 2 & 3 & : & 5 & 2 & 5 & 3 & 4 \\
\hline$n$ & 22 & 23 & 24 & 25 & 26 & 27 & & 28 & 29 & 30 & 31 & 32 & 33 & 34 & 35 & 36 & 37 & 38 \\
\hline$I(n)$ & 11 & 6 & 28 & 10 & 14 & 13 & & 21 & 8 & 35 & 8 & 22 & 17 & 18 & 17 & 41 & 10 & 19 \\
\hline$I_{c}(n)$ & 8 & 6 & 14 & 8 & 10 & 9 & & 13 & 8 & 19 & 8 & 12 & 13 & 13 & 13 & 19 & 10 & 14 \\
\hline$P(n)$ & 8 & 6 & 11 & 8 & 10 & 9 & & 11 & 8 & 13 & 8 & 12 & 12 & 13 & 12 & 15 & 10 & 14 \\
\hline$P_{r}(n)$ & 3 & 6 & 4 & 6 & 4 & 5 & & 4 & 8 & 3 & 8 & 5 & 6 & 5 & 7 & 4 & 10 & 5 \\
\hline$n$ & 39 & 40 & 41 & 42 & 43 & 44 & & 45 & 46 & 47 & 48 & 49 & 50 & 51 & 52 & 53 & 54 & 55 \\
\hline$I(n)$ & 20 & 40 & 11 & 44 & 11 & 31 & & 32 & 23 & 12 & 60 & 16 & 36 & 25 & 37 & 14 & 49 & 24 \\
\hline$I_{c}(n)$ & 15 & 20 & 11 & 25 & 11 & 19 & & 19 & 17 & 12 & 26 & 14 & 22 & 19 & 22 & 14 & 26 & 19 \\
\hline$P(n)$ & 14 & 17 & 11 & 18 & 11 & 17 & & 17 & 17 & 12 & 21 & 14 & 20 & 18 & 20 & 14 & 22 & 18 \\
\hline$P_{r}(n)$ & 7 & 6 & 11 & 4 & 11 & 6 & & 7 & 6 & 12 & 6 & 11 & 6 & 9 & 7 & 14 & 5 & 11 \\
\hline$n$ & 56 & 57 & 58 & 59 & 60 & 61 & & 62 & 63 & 64 & 65 & 66 & 67 & 68 & 69 & 70 & 71 & 72 \\
\hline$I(n)$ & 50 & 27 & 30 & 15 & 93 & 16 & & 31 & 40 & 46 & 29 & 64 & 17 & 47 & 32 & 63 & 18 & 96 \\
\hline$I_{c}(n)$ & 26 & 21 & 22 & 15 & 40 & 16 & & 23 & 25 & 24 & 23 & 37 & 17 & 28 & 25 & 37 & 18 & 38 \\
\hline$P(n)$ & 23 & 20 & 22 & 15 & 27 & 16 & & 23 & 23 & 24 & 22 & 28 & 17 & 26 & 24 & 29 & 18 & 31 \\
\hline$P_{r}(n)$ & 8 & 10 & 8 & 15 & 6 & 16 & & 8 & 10 & 9 & 14 & 6 & 17 & 9 & 12 & 7 & 18 & 8 \\
\hline$n$ & 73 & 74 & 75 & 76 & 77 & & 78 & 79 & 80 & 81 & 82 & 83 & & 84 & 85 & 87 & 88 & \\
\hline$I(n)$ & 19 & 38 & 49 & 51 & 30 & & 75 & 20 & 84 & 40 & 42 & 21 & & 17 & 36 & 40 & $7 \%$ & \\
\hline$I_{c}(n)$ & 19 & 28 & 31 & 31 & 2 & & 43 & 20 & 38 & 27 & 31 & 21 & & 52 & 29 & 31 & 38 & \\
\hline$P(n)$ & 19 & 28 & 28 & 29 & 2 & & 33 & 20 & 33 & 27 & 31 & 21 & & 37 & 28 & 30 & 3 & \\
\hline$P_{r}(n)$ & 19 & 10 & 11 & 10 & 16 & & 7 & 20 & 10 & 14 & 11 & 21 & & 8 & 18 & 15 & 12 & \\
\hline$n$ & 89 & 90 & 91 & & 92 & 3 & 94 & 95 & 96 & 97 & & 98 & 99 & 100 & 101 & 102 & 10 & \\
\hline$I(n)$ & 23 & 120 & 35 & & 61 & 22 & 47 & 38 & 122 & 25 & & 62 & 57 & 93 & 26 & 95 & 26 & \\
\hline$I_{c}(n)$ & 23 & 55 & 29 & 3 & 37 & 33 & 35 & 31 & 50 & 25 & & 41 & 37 & 46 & 26 & 55 & 2 & \\
\hline$P(n)$ & 23 & 39 & 28 & 3 & 35 & 2 & 35 & 30 & 41 & 25 & & 38 & 35 & 40 & 26 & 43 & 2 & \\
\hline$P_{r}(n)$ & 23 & 7 & 19 & & 12 & 6 & 12 & 19 & 10 & 25 & & 11 & 16 & 11 & 26 & 9 & 2 & \\
\hline
\end{tabular}

Table 2: The values of $I(n), I_{c}(n), P(n), P_{r}(n)$ for $3 \leq n \leq 103$. 


\begin{tabular}{c|ccccccccccccc}
$n$ & 104 & 105 & 106 & 107 & 108 & 109 & 110 & 111 & 112 & 113 & 114 & 115 & 116 \\
\hline$I(n)$ & 84 & 85 & 54 & 27 & 131 & 28 & 91 & 50 & 106 & 29 & 104 & 45 & 77 \\
$I_{c}(n)$ & 44 & 51 & 40 & 27 & 55 & 28 & 55 & 39 & 50 & 29 & 61 & 37 & 46 \\
$P(n)$ & 41 & 42 & 40 & 27 & 45 & 28 & 45 & 38 & 45 & 29 & 48 & 36 & 44 \\
$P_{r}(n)$ & 14 & 14 & 14 & 27 & 10 & 28 & 11 & 19 & 14 & 29 & 10 & 23 & 15 \\
$n$ & 117 & 118 & 119 & 120 & 121 & 122 & 123 & 124 & 125 & 126 & 127 & 128 & 129 \\
\hline$I(n)$ & 66 & 59 & 44 & 208 & 36 & 62 & 55 & 81 & 48 & 153 & 32 & 94 & 57 \\
$I_{c}(n)$ & 43 & 44 & 37 & 78 & 33 & 46 & 43 & 49 & 38 & 73 & 32 & 48 & 45 \\
$P(n)$ & 41 & 44 & 36 & 55 & 33 & 46 & 42 & 47 & 38 & 54 & 32 & 48 & 44 \\
$P_{r}(n)$ & 19 & 15 & 25 & 12 & 28 & 16 & 21 & 16 & 26 & 10 & 32 & 17 & 22 \\
$n$ & 130 & 131 & 132 & 133 & 134 & 135 & 136 & 137 & 138 & 139 & 140 & 141 & 142 \\
\hline$I(n)$ & 108 & 33 & 167 & 48 & 67 & 96 & 106 & 35 & 124 & 35 & 163 & 62 & 71 \\
$I_{c}(n)$ & 65 & 33 & 76 & 41 & 50 & 55 & 56 & 35 & 73 & 35 & 76 & 49 & 53 \\
$P(n)$ & 54 & 33 & 57 & 40 & 50 & 50 & 53 & 35 & 58 & 35 & 59 & 48 & 53 \\
$P_{r}(n)$ & 14 & 33 & 12 & 28 & 17 & 19 & 18 & 35 & 12 & 35 & 14 & 24 & 18
\end{tabular}

Table 3: The values of $I(n), I_{c}(n), P(n), P_{r}(n)$ for $105 \leq n \leq 142$.

\begin{tabular}{c|ccccccccccccccccc}
$n$ & 4 & 6 & 8 & 10 & 12 & 14 & 16 & 18 & 20 & 22 & 24 & 26 & 28 & 30 & 32 & 34 & 36 \\
\hline$I_{b c}(n)$ & 1 & 1 & 2 & 2 & 3 & 2 & 3 & 3 & 4 & 3 & 6 & 4 & 5 & 7 & 5 & 5 & 7 \\
$P_{b}(n)$ & 1 & 1 & 2 & 2 & 3 & 2 & 3 & 3 & 4 & 3 & 6 & 4 & 5 & 6 & 5 & 5 & 7 \\
$n$ & 38 & 40 & 42 & 44 & 46 & 48 & 50 & 52 & 54 & 56 & 58 & 60 & 62 & 64 & 66 & 68 \\
\hline$I_{b c}(n)$ & 5 & 8 & 9 & 7 & 6 & 10 & 8 & 8 & 9 & 10 & 8 & 14 & 8 & 9 & 13 & 10 \\
$P_{b}(n)$ & 5 & 8 & 8 & 7 & 6 & 10 & 8 & 8 & 9 & 10 & 8 & 13 & 8 & 9 & 12 & 10 \\
$n$ & 70 & 72 & 74 & 76 & 78 & 80 & 82 & 84 & 86 & 88 & 90 & 92 & 94 & 96 & 98 & 100 \\
\hline$I_{b c}(n)$ & 13 & 14 & 10 & 11 & 15 & 14 & 11 & 18 & 11 & 14 & 19 & 13 & 12 & 18 & 14 & 16 \\
$P_{b}(n)$ & 12 & 14 & 10 & 11 & 14 & 14 & 11 & 17 & 11 & 14 & 17 & 13 & 12 & 18 & 14 & 16 \\
$n$ & 102 & 104 & 106 & 108 & 110 & 112 & 114 & 116 & 118 & 120 & 122 & 124 & 126 \\
\hline$I_{b c}(n)$ & 19 & 16 & 14 & 19 & 19 & 18 & 21 & 16 & 15 & 28 & 16 & 17 & 25 \\
$P_{b}(n)$ & 18 & 16 & 14 & 19 & 18 & 18 & 20 & 16 & 15 & 26 & 16 & 17 & 23 \\
$n$ & 128 & 130 & 132 & 134 & 136 & 138 & 140 & 142 & 144 & 146 & 148 & 150 & 152 \\
\hline$I_{b c}(n)$ & 17 & 23 & 26 & 17 & 20 & 25 & 26 & 18 & 26 & 19 & 20 & 31 & 22 \\
$P_{b}(n)$ & 17 & 22 & 25 & 17 & 20 & 24 & 25 & 18 & 26 & 19 & 20 & 28 & 22
\end{tabular}

Table 4: The values of $I_{b c}(2 n)$ and $P_{b}(2 n)$ for $2 \leq n \leq 76$. 


\section{The proofs}

\subsection{The Burnside technology}

Let $\alpha$ be the action of a finite group $G$ on a finite set $A$. Then we denote by $\sim_{\alpha}$ the associated equivalence relation on $A$, by $\left|A / \sim_{\alpha}\right|$ the number of orbits of $\alpha$, and by fix $x_{\alpha}(g)$ the number of elements of $A$ fixed by $g \in G$ under $\alpha$. Our main enumeration tool is the Cauchy-Frobenius-Burnside lemma:

\section{Lemma 3.1.}

$$
\left|A / \sim_{\alpha}\right|=\frac{1}{|G|} \sum_{g \in G} \operatorname{fix}_{\alpha}(g) .
$$

For a proof, see, e.g., [11, Lemma 7.24.5]).

First we list some auxiliary results which will be useful in the sequel.

Proposition 3.2. Let $\vartheta_{n}$ be the multiplicative action of $\mathbb{Z}_{n}^{*}$ on $\mathbb{Z}_{n}$. Then

$$
\left|\mathbb{Z}_{n} / \sim_{\vartheta_{n}}\right|=\frac{1}{\varphi(n)} \sum_{a \in \mathbb{Z}_{n}^{*}} \operatorname{gcd}(n, a-1) .
$$

Proof. Assume that $j \in \mathbb{Z}_{n}, a \in \mathbb{Z}_{n}^{*}, d=\operatorname{gcd}(n, a-1), n=n^{\prime} d$ and $a-1=a^{\prime} d$. Then $\operatorname{gcd}\left(n^{\prime}, a^{\prime}\right)=1$, and so $j$ is fixed by $a$ iff

$$
a j \equiv j \quad(\bmod n) \Longleftrightarrow n\left|(a-1) j \Longleftrightarrow n^{\prime}\right| a^{\prime} j \Longleftrightarrow n^{\prime} \mid j .
$$

It follows that the set of $j$ fixed by $a$ is $\left\{0, n^{\prime}, 2 n^{\prime}, \ldots,(d-1) n^{\prime}\right\}$, hence $\operatorname{fix}_{\vartheta}(a)=d=$ $\operatorname{gcd}(n, a-1)$, and Lemma 3.1 gives (3.1).

Lemma 3.3. Let $a, d, n \in \mathbb{N}$ be such that $d \mid n$ and $\operatorname{gcd}(a, d)=1$. Then there is an $x \in \mathbb{Z}$ such that $\operatorname{gcd}(a+x d, n)=1$.

Proof. Let $x \in \mathbb{Z}_{n}$ satisfy

$$
x \not \equiv-a d^{-1}(\bmod p)
$$

for each prime $p$ which divides $n$ but not $d$. Note that $d$ is invertible $\bmod p$ for such $p$, and that such an $x$ exists by the Chinese Remainder Theorem.

Assume that $\operatorname{gcd}(a+x d, n) \neq 1$. Then there exists a prime $p$ such that $p \mid n$ and $p \mid(a+x d)$. We distinguish two cases.

a) If $p \mid d$ then $p \mid a$, contrary to the assumption that $\operatorname{gcd}(a, d)=1$.

b) If $p \nmid d$ then

$$
a+x d \equiv 0(\bmod p) \Longrightarrow x \equiv-a d^{-1}(\bmod p),
$$

contrary to the choice of $x$.

In either case we reach a contradiction, hence $\operatorname{gcd}(a+x d, n)=1$.

Corollary 3.4. Let $\vartheta_{n}$ be as in Proposition 3.2. For all $j, k \in \mathbb{Z}_{n}$ we have:

(i) $j \sim_{\vartheta_{n}} \operatorname{gcd}(n, j)$, 
(ii) $j \sim_{\vartheta_{n}} k \Longleftrightarrow \operatorname{gcd}(n, j)=\operatorname{gcd}(n, k)$,

(iii) each orbit of $\vartheta_{n}$ contains exactly one positive divisor of $n$ (with $n$ replaced by 0 ), and $\left|\mathbb{Z}_{n} / \sim_{\vartheta_{n}}\right|=\tau(n)$.

Proof. (i) Let $d=\operatorname{gcd}(n, j), n^{\prime}=n / d, j^{\prime}=j / d$. Then $\operatorname{gcd}\left(n^{\prime}, j^{\prime}\right)=1$, so there are $a^{\prime}, k \in \mathbb{Z}$ such that $a^{\prime} j^{\prime}=1+k n^{\prime}$. Since $\operatorname{gcd}\left(a^{\prime}, n^{\prime}\right)=1$ and $n^{\prime} \mid n$, Lemma 3.3 implies that there is an $x \in \mathbb{Z}$ such that $a:=a^{\prime}+x n^{\prime} \in \mathbb{Z}_{n}^{*}$. Then

$$
a j=\left(a^{\prime}+x n^{\prime}\right) j^{\prime} d=a^{\prime} j^{\prime} d+x j^{\prime} n=\left(1+k n^{\prime}\right) d+x j^{\prime} n=d+\left(k+x j^{\prime}\right) n,
$$

hence $a j \equiv d(\bmod n)$. So $j \sim_{\vartheta_{n}} d$, proving the claim.

(ii) Let $j \sim_{\vartheta_{n}} k$. Then there are $a \in \mathbb{Z}_{n}^{*}$ and $m \in \mathbb{Z}$ such that $a j-k=m n$. This implies that any common divisor of $j$ and $n$ divides $k$, and any common divisor of $k$ and $n$ divides $a j$ and hence $j$. It follows that $\operatorname{gcd}(n, j)=\operatorname{gcd}(n, k)$.

Conversely, let $\operatorname{gcd}(n, j)=\operatorname{gcd}(n, k)$. Then by (i), $j \sim_{\vartheta_{n}} k$.

(iii) By (i), each orbit of $\sim_{\vartheta_{n}}$ contains a positive divisor of $n$ (with $n$ replaced by 0 ). By (ii), different positive divisors of $n$ (with $n$ replaced by 0 ) belong to different orbits of $\sim_{\vartheta_{n}}$. This proves the claim.

Lemma 3.5. Let $a, b, c \in \mathbb{Z}, n, k \in \mathbb{N}$.

(i) If $a \equiv b(\bmod n)$ then $\operatorname{gcd}(a, n)=\operatorname{gcd}(b, n)$.

(ii) If $\operatorname{gcd}(a, b)=1$ then $\operatorname{gcd}(a b, c)=\operatorname{gcd}(a, c) \operatorname{gcd}(b, c)$.

(iii) Any set of $n k$ consecutive integers contains exactly $k$ multiples of $n$.

The straightforward proofs are omitted.

Now we embark on our main task of enumerating isomorphism classes of I-graphs. For a fixed $n \geq 3$, we represent the I-graph $I(n, j, k)$ with the ordered pair $(j, k)$. We need to construct a suitable group $G_{n}$ acting on the set $\mathbb{Z}_{n} \times \mathbb{Z}_{n}$ in such a way that the orbits of this action will be in one-to-one correspondence with the isomorphism classes of I-graphs. In view of Theorem 1.1, the following choice is natural.

Definition 3.6. By $G_{n}$ we denote the subgroup of the symmetric group $S\left(\mathbb{Z}_{n} \times \mathbb{Z}_{n}\right)$ generated by the permutations $\left(\xi_{a}\right)_{a \in \mathbb{Z}_{n}^{*}}, \mu, \rho: \mathbb{Z}_{n} \times \mathbb{Z}_{n} \rightarrow \mathbb{Z}_{n} \times \mathbb{Z}_{n}$, where for all $a \in \mathbb{Z}_{n}^{*}$ and $(j, k) \in \mathbb{Z}_{n} \times \mathbb{Z}_{n}$ :

$$
\begin{aligned}
\xi_{a}(j, k) & \equiv(a j, a k) \quad(\bmod n), \\
\mu(j, k) & \equiv(j,-k) \quad(\bmod n), \\
\rho(j, k) & \equiv(k, j) \quad(\bmod n) .
\end{aligned}
$$

\section{Proposition 3.7.}

$$
G_{n}=\left\{\xi_{a}, \xi_{a} \mu, \xi_{a} \rho, \xi_{a} \rho \mu ; a \in \mathbb{Z}_{n}^{*}\right\}
$$

and $\left|G_{n}\right|=4 \varphi(n)$.

Proof. It is straightforward to check that for all $a, b \in \mathbb{Z}_{n}^{*}$,

$$
\begin{aligned}
\xi_{a} \xi_{b} & =\xi_{a b}, \\
\xi_{a} \xi_{a^{-1}}=\xi_{1} & =\operatorname{id}_{\mathbb{Z}_{n} \times \mathbb{Z}_{n}}=\mu^{2}=\rho^{2}, \\
\mu \xi_{a} & =\xi_{a} \mu, \\
\rho \xi_{a} & =\xi_{a} \rho, \\
\mu \rho & =\xi_{-1} \rho \mu .
\end{aligned}
$$


Using these equalities we can show that for any $g \in G_{n}$ there are $a \in \mathbb{Z}_{n}^{*}$ and $\epsilon, \delta \in\{0,1\}$ such that

$$
g=\xi_{a} \rho^{\epsilon} \mu^{\delta}
$$

which proves (3.2). Now write $g_{i}=\xi_{a_{i}} \rho^{\epsilon_{i}} \mu^{\delta_{i}}$ for $i \in\{1,2\}$. Assume that $g_{1}=g_{2}$, and compute

$$
g_{i}(1,1)= \begin{cases}\left(a_{i},(-1)^{\delta_{i}} a_{i}\right), & \epsilon_{i}=0, \\ \left((-1)^{\delta_{i}} a_{i}, a_{i}\right), & \epsilon_{i}=1 .\end{cases}
$$

If $\epsilon_{1} \neq \epsilon_{2}$, then $g_{1}(1,1)=g_{2}(1,1)$ implies that $a_{1}=(-1)^{\delta_{2}} a_{2}$ and $a_{2}=(-1)^{\delta_{1}} a_{1}$, hence $a_{1}=(-1)^{\delta_{1}+\delta_{2}} a_{1}$. Cancelling $a_{1}$ yields $(-1)^{\delta_{1}+\delta_{2}}=1$, and so $\delta_{1}=\delta_{2}$. W.l.g. assume that $\epsilon_{1}=1$ and $\epsilon_{2}=0$. Then $g_{1}=g_{2}$ turns into $\xi_{a_{1}} \rho=\xi_{a_{2}}$. Applying both sides of this equality to $(1,1)$ yields $\left(a_{1}, a_{1}\right)=\left(a_{2}, a_{2}\right)$, hence $a_{1}=a_{2}$ and $\xi_{a_{1}}=\xi_{a_{2}}$. Now $\xi_{a_{1}} \rho=\xi_{a_{2}}$ implies $\rho=\xi_{1}$. On the other hand, the initial assumption that $n \geq 3$ implies that $\left|\mathbb{Z}_{n}^{*}\right| \geq 2$, hence $\rho \neq \xi_{1}$.

This contradiction shows that $\epsilon_{1}=\epsilon_{2}$. Then $g_{1}(1,1)=g_{2}(1,1)$ implies that $a_{1}=a_{2}$ and $(-1)^{\delta_{1}} a_{1}=(-1)^{\delta_{2}} a_{2}$, hence $(-1)^{\delta_{1}}=(-1)^{\delta_{2}}$, and so $\delta_{1}=\delta_{2}$.

We have shown that $g_{1}=g_{2}$ if and only if $a_{1}=a_{2}$ and $\epsilon_{1}=\epsilon_{2}$ and $\delta_{1}=\delta_{2}$. Hence $\left|G_{n}\right|=4\left|\mathbb{Z}_{n}^{*}\right|=4 \varphi(n)$ as claimed.

Remark 3.8. Let $\langle\rho, \mu\rangle$ be the subgroup of $G_{n}$ generated by $\rho$ and $\mu$. One can see that $\langle\rho, \mu\rangle=\left\{\xi_{1}, \rho, \mu, \rho \mu, \xi_{-1}, \xi_{-1} \rho, \xi_{-1} \mu, \xi_{-1} \rho \mu\right\}$ is isomorphic to the dihedral group $D_{4}=$ $\left\langle r, s \mid r^{4}=f^{2}=(r f)^{2}=1\right\rangle$, with $r$ corresponding to $\rho \mu$ or $\mu \rho$, and $f$ corresponding to any of $\rho, \mu, \rho \mu \rho$, or $\mu \rho \mu$. The mapping $h: \mathbb{Z}_{n}^{*} \times D_{4} \rightarrow G_{n}$ defined by

$$
h\left(a, r^{i} f^{j}\right)=\xi_{a}(\rho \mu)^{i} \rho^{j}, \quad \text { for } i \in\{0,1,2,3\}, j \in\{0,1\},
$$

is a group epimorphism with kernel $C_{2}=\left\langle\left(-1, r^{2}\right)\right\rangle$, hence by the first isomorphism theorem for groups, $G_{n} \simeq\left(\mathbb{Z}_{n}^{*} \times D_{4}\right) / C_{2}$.

The elements of $G_{n}$ are permutations of $\mathbb{Z}_{n} \times \mathbb{Z}_{n}$, hence the group $G_{n}$ acts naturally on $\mathbb{Z}_{n} \times \mathbb{Z}_{n}$. We denote this action by $\alpha_{n}$. In the next lemma we show how to count the isomorphism classes in a set $\mathcal{K}_{n}$ of I-graphs on $2 n$ vertices, by counting the orbits of $\alpha_{n}$ on an appropriate subset $K_{n} \subseteq \mathbb{Z}_{n} \times \mathbb{Z}_{n}$.

Lemma 3.9. Let $\mathcal{K}_{n} \subseteq\left\{I(n, j, k) ; j, k \in \mathbb{Z}_{n}^{\prime}\right\}$ be a set of I-graphs closed under isomorphism. Let $K_{n} \subseteq \mathbb{Z}_{n} \times \mathbb{Z}_{n}$ satisfy

$$
K_{n} \cap\left(\mathbb{Z}_{n}^{\prime} \times \mathbb{Z}_{n}^{\prime}\right)=\left\{(j, k) ; I(n, j, k) \in \mathcal{K}_{n}\right\},
$$

and $g\left(K_{n}\right)=K_{n}$ for all $g \in G_{n}$. Then the restriction of $G_{n}$ to $K_{n}$,

$$
\left.G\right|_{K_{n}}:=\left\{\left.g\right|_{K_{n}} ; g \in G_{n}\right\},
$$

is a subgroup of $S\left(K_{n}\right)$, so let $\alpha\left(K_{n}\right)$ be the action of $\left.G\right|_{K_{n}}$ on $K_{n}$. Write

$$
\nu_{0}\left(K_{n}\right)=\left|\left\{\eta \in K_{n} / \sim_{\alpha\left(K_{n}\right)} ; \eta \nsubseteq \mathbb{Z}_{n}^{\prime} \times \mathbb{Z}_{n}^{\prime}\right\}\right|
$$

Then

$$
\left|\mathcal{K}_{n} / \simeq\right|=\left|K_{n} / \sim_{\alpha\left(K_{n}\right)}\right|-\nu_{0}\left(K_{n}\right)
$$

where $\simeq$ denotes graph isomorphism. 
Proof. Let us write $K_{n}^{\prime}=\left\{(j, k) \in \mathbb{Z}_{n}^{\prime} \times \mathbb{Z}_{n}^{\prime} ; I(n, j, k) \in \mathcal{K}_{n}\right\}$. Note that for any $(j, k)$, $\left(j^{\prime}, k^{\prime}\right) \in \mathbb{Z}_{n}^{\prime} \times \mathbb{Z}_{n}^{\prime}$ we have, by Theorem 1.1 and Proposition 3.7,

$$
\begin{aligned}
& I(n, j, k) \simeq I\left(n, j^{\prime}, k^{\prime}\right) \\
& \quad \Longleftrightarrow \exists a \in \mathbb{Z}_{n}^{*}:\left\{j^{\prime}, k^{\prime}\right\} \in\{\{a j, a k\},\{a j,-a k\}\} \\
& \quad \Longleftrightarrow \exists a \in \mathbb{Z}_{n}^{*}:\left(j^{\prime}, k^{\prime}\right) \in\{(a j, a k),(a k, a j),(a j,-a k),(-a k, a j)\} \\
& \quad \Longleftrightarrow \exists a \in \mathbb{Z}_{n}^{*}:\left(j^{\prime}, k^{\prime}\right) \in\left\{\xi_{a}(j, k), \xi_{a} \rho(j, k), \xi_{a} \mu(j, k), \xi_{a} \rho \mu(j, k)\right\} \\
& \quad \Longleftrightarrow \exists g \in G_{n}:\left(j^{\prime}, k^{\prime}\right)=g(j, k)
\end{aligned}
$$

where all the arithmetic is done modulo $n$.

Let $(j, k) \in K_{n}^{\prime}$ and $\left(j^{\prime}, k^{\prime}\right)=g(j, k)$ for some $g \in G_{n}$. Then $I(n, j, k) \in \mathcal{K}_{n}$, and $I(n, j, k) \simeq I\left(n, j^{\prime}, k^{\prime}\right)$ by (3.4), hence $I\left(n, j^{\prime}, k^{\prime}\right) \in \mathcal{K}_{n}$ and $\left(j^{\prime}, k^{\prime}\right) \in K_{n}^{\prime}$. It follows that $g\left(K_{n}^{\prime}\right)=K_{n}^{\prime}$ for all $g \in G_{n}$, so $\left.G\right|_{K_{n}^{\prime}}$ is a subgroup of $S\left(K_{n}^{\prime}\right)$. Let $\alpha\left(K_{n}^{\prime}\right)$ be the action of $\left.G\right|_{K_{n}^{\prime}}$ on $K_{n}^{\prime}$. By Theorem 1.1, the mapping

$$
f:[I(n, j, k)] \mapsto[(j, k)]
$$

from $\mathcal{K}_{n} / \simeq$ to $K_{n}^{\prime} / \sim_{\alpha\left(K_{n}^{\prime}\right)}$ is well defined and injective. Obviously it is also surjective, hence

$$
\left|\mathcal{K}_{n} / \simeq\right|=\left|K_{n}^{\prime} / \sim_{\alpha\left(K_{n}^{\prime}\right)}\right|
$$

We claim that for any orbit $\eta \in K_{n} / \sim_{\alpha\left(K_{n}\right)}$, either $\eta \subseteq \mathbb{Z}_{n}^{\prime} \times \mathbb{Z}_{n}^{\prime}$ or $\eta \subseteq\left(\mathbb{Z}_{n} \times \mathbb{Z}_{n}\right) \backslash$ $\left(\mathbb{Z}_{n}^{\prime} \times \mathbb{Z}_{n}^{\prime}\right)$. To prove this, assume that $\eta \nsubseteq \mathbb{Z}_{n}^{\prime} \times \mathbb{Z}_{n}^{\prime}$. Then $(0, k) \in \eta$ or $(n / 2, k) \in \eta$ for some $k \in \mathbb{Z}_{n}$ (the latter only if $n$ is even). Hence for any $\left(j^{\prime}, k^{\prime}\right) \in \eta$, there is a $g \in G_{n}$ such that $\left(j^{\prime}, k^{\prime}\right) \in\{g(0, k), g(n / 2, k)\}$. From Proposition 3.7 it follows that there are $a, b, c \in \mathbb{Z}_{n}^{*}$ such that $\left\{j^{\prime}, k^{\prime}\right\} \in\{\{0, a k\},\{b n / 2, c k\}\}$. If $n$ is even then $b$ is odd, hence $n \mid n(b-1) / 2$ and $b n / 2 \equiv n / 2(\bmod n)$, implying that $\left\{j^{\prime}, k^{\prime}\right\} \in\{\{0, a k\},\{n / 2, c k\}\}$ for some $a, c \in \mathbb{Z}_{n}^{*}$. We conclude that $\eta \subseteq\left(\mathbb{Z}_{n} \times \mathbb{Z}_{n}\right) \backslash\left(\mathbb{Z}_{n}^{\prime} \times \mathbb{Z}_{n}^{\prime}\right)$ which proves the claim.

It follows that every orbit of $\alpha\left(K_{n}^{\prime}\right)$ is an orbit of $\alpha\left(K_{n}\right)$, and every orbit of $\alpha\left(K_{n}\right)$ is either an orbit of $\alpha\left(K_{n}^{\prime}\right)$ or is contained in $\left(\mathbb{Z}_{n} \times \mathbb{Z}_{n}\right) \backslash\left(\mathbb{Z}_{n}^{\prime} \times \mathbb{Z}_{n}^{\prime}\right)$. Hence

$$
\left|K_{n} / \sim_{\alpha\left(K_{n}\right)}\right|=\left|K_{n}^{\prime} / \sim_{\alpha\left(K_{n}^{\prime}\right)}\right|+\nu_{0}\left(K_{n}\right),
$$

which, together with (3.5), completes the proof.

In the rest of the paper we proceed as follows. For each of the (five) sets $\mathcal{K}_{n}$ of Igraphs whose isomorphism classes we wish to enumerate, we select an appropriate set $K_{n} \subseteq \mathbb{Z}_{n} \times \mathbb{Z}_{n}$, and check that the assumptions of Lemma 3.9 are satisfied. Then we count the orbits of $\alpha\left(K_{n}\right)$ by means of Lemma 3.1, which is tantamount to computing the average number of fixed points of the elements $\left.g \in G\right|_{K_{n}}$. This is done by counting the fixed points of $g$ in four steps, corresponding to the four possible types of $g$, namely $\xi_{a}$, $\xi_{a} \mu, \xi_{a} \rho$ and $\xi_{a} \rho \mu$ (with $a \in \mathbb{Z}_{n}^{*}$ ). Finally we compute $\nu_{0}\left(K_{n}\right)$ by counting those orbits of $\alpha\left(K_{n}\right)$ that contain an element of the form $(0, k)$ or $(n / 2, k)$, and use (3.3).

To simplify notation, we write $G_{n}$ for $\left.G\right|_{K_{n}}$ and $\alpha_{n}$ for $\alpha\left(K_{n}\right)$ in the sequel. This causes no confusion, since in each of the five cases considered it is straightforward to verify that $\left.G\right|_{K_{n}} \simeq G_{n}$. 


\subsection{I-graphs}

Let $\mathcal{K}_{n}$ be the set of all I-graphs on $2 n$ vertices, and $K_{n}:=\mathbb{Z}_{n} \times \mathbb{Z}_{n}$.

\section{Proposition 3.10.}

$$
\left|\mathbb{Z}_{n} \times \mathbb{Z}_{n} / \sim_{\alpha_{n}}\right|=\frac{1}{4 \varphi(n)} \sum_{i=1}^{4} \sum_{a \in \mathbb{Z}_{n}^{*}} f_{i}(a, n)
$$

where

$$
\begin{aligned}
& f_{1}(a, n)=\operatorname{gcd}(n, a-1)^{2}, \\
& f_{2}(a, n)=\operatorname{gcd}(n, a-1) \operatorname{gcd}(n, a+1) \\
& f_{3}(a, n)=\operatorname{gcd}\left(n, a^{2}-1\right) \\
& f_{4}(a, n)=\operatorname{gcd}\left(n, a^{2}+1\right) .
\end{aligned}
$$

Proof. We use Lemma 3.1. The fixed points of $\xi_{a}$ are those pairs $(j, k)$ which satisfy $a j \equiv j(\bmod n)$ and $a k \equiv k(\bmod n)$. As in the proof of Proposition 3.2 we see that there are $d=\operatorname{gcd}(n, a-1) \operatorname{such} j$ 's, and $d$ such $k$ 's, hence $d^{2}$ such pairs. The number of fixed points of all $\xi_{a}$ is thus $\sum_{a \in \mathbb{Z}_{n}^{*}} f_{1}(a, n)$.

The fixed points of $\xi_{a} \mu$ are those pairs $(j, k)$ which satisfy $a j \equiv j(\bmod n)$ and $-a k \equiv$ $k(\bmod n)$. There are $\operatorname{gcd}(n, a-1) \operatorname{such} j$ 's, and $\operatorname{gcd}(n, a+1) \operatorname{such} k$ 's, hence the number of fixed points of all $\xi_{a} \mu$ is $\sum_{a \in \mathbb{Z}_{n}^{*}} f_{2}(a, n)$.

The fixed points of $\xi_{a} \rho$ are those pairs $(j, k)$ which satisfy $a k \equiv j(\bmod n)$ and $a j \equiv k(\bmod n)$. Hence $a^{2} k \equiv k(\bmod n)$, and for any such $k$, we must take $j \equiv a k$ $(\bmod n)$. There are $\operatorname{gcd}\left(n, a^{2}-1\right) \operatorname{such} k$ 's, hence the number of fixed points of all $\xi_{a} \rho$ is $\sum_{a \in \mathbb{Z}_{n}^{*}} f_{3}(a, n)$.

The fixed points of $\xi_{a} \rho \mu$ are those pairs $(j, k)$ which satisfy $-a k \equiv j(\bmod n)$ and $a j \equiv k(\bmod n)$. Hence $-a^{2} k \equiv k(\bmod n)$, and for any such $k$, we must take $j \equiv-a k$ $(\bmod n)$. There are $\operatorname{gcd}\left(n, a^{2}+1\right) \operatorname{such} k$ 's, hence the number of fixed points of all $\xi_{a} \rho \mu$ is $\sum_{a \in \mathbb{Z}_{n}^{*}} f_{4}(a, n)$.

Since $\left|G_{n}\right|=4 \varphi(n)$, the assertion follows.

Now we wish to evaluate the sum appearing in Proposition 3.10 in closed form, given the prime factorization of $n$. We do this by splitting this double sum into four single sums correspondng to $i=1,2,3,4$, evaluating each of them in the case when $n$ is a prime power, and showing that they are multiplicative.

Lemma 3.11. For $i=1,2,3,4$, let

$$
g_{i}(n)=\frac{1}{\varphi(n)} \sum_{a \in \mathbb{Z}_{n}^{*}} f_{i}(a, n)
$$

where $f_{i}(a, n)$ are as in Proposition 3.10. If $p$ is a prime and $k \geq 1$, then $g_{i}\left(p^{k}\right)$ are as given in equations (2.2) - (2.5). 
Proof. Let $x, r \in \mathbb{Z}$ with $\operatorname{gcd}(r, p)=1$. Denote

$$
\begin{aligned}
\nu_{p}(x) & =\max \left\{i \in \mathbb{N} ; p^{i} \mid x\right\} \\
M_{k, j}^{(r)}(p) & =\left\{x \in \mathbb{Z}_{p^{k}}^{*}-r ; \nu_{p}(x) \geq j\right\}, \text { for } 1 \leq j \leq k, \\
N_{k, j}^{(r)}(p) & =\left\{x \in \mathbb{Z}_{p^{k}}^{*}-r ; \nu_{p}(x)=j\right\}, \text { for } 0 \leq j \leq k-1 .
\end{aligned}
$$

The elements of $\left(\mathbb{Z}_{p^{k}} \backslash \mathbb{Z}_{p^{k}}^{*}\right)-r$ are not divisible by $p$, hence it follows for $j \geq 1$ that $M_{k, j}^{(r)}(p)=\left\{x \in \mathbb{Z}_{p^{k}}-r ; \nu_{p}(x) \geq j\right\}$. This is the set of all multiples of $p^{j}$ in a set of $p^{k}$ consecutive integers, therefore Lemma 3.5 (iii) implies that $\left|M_{k, j}^{(r)}(p)\right|=p^{k-j}$ for $1 \leq j \leq k$ and for all $r$ such that $\operatorname{gcd}(r, p)=1$. Consequently

$$
\begin{aligned}
\left|N_{k, j}^{(r)}(p)\right| & =\left|M_{k, j}^{(r)}(p)\right|-\left|M_{k, j+1}^{(r)}(p)\right|=p^{k-j}-p^{k-j-1} \quad \text { for } 1 \leq j \leq k-1 \\
\left|N_{k, 0}^{(r)}(p)\right| & =\left|\mathbb{Z}_{p^{k}}^{*}-r\right|-\left|M_{k, 1}^{(r)}(p)\right|=\varphi\left(p^{k}\right)-p^{k-1}=p^{k}-2 p^{k-1}
\end{aligned}
$$

It follows that for any $s \in \mathbb{N}$ we have

$$
\begin{gathered}
\sum_{a \in \mathbb{Z}_{p^{*}}^{*}} \operatorname{gcd}\left(p^{k}, a-r\right)^{s}=\sum_{j=0}^{k-1}\left|N_{k, j}^{(r)}(p)\right| p^{s j}+\left|M_{k, k}^{(r)}(p)\right| p^{s k} \\
=p^{k}-2 p^{k-1}+p^{k} \sum_{j=1}^{k-1}\left(p^{(s-1) j}-p^{(s-1) j-1}\right)+p^{s k}
\end{gathered}
$$

which for $s=1$ turns into

$$
\sum_{a \in \mathbb{Z}_{p^{k}}^{*}} \operatorname{gcd}\left(p^{k}, a-r\right)=(k+1) \varphi\left(p^{k}\right) .
$$

Now we compute $g_{i}\left(p^{k}\right)$ for $i=1,2,3,4$.

(i) By (3.6) with $r=1$ and $s=2$ we have

$$
g_{1}\left(p^{k}\right) \varphi\left(p^{k}\right)=\sum_{a \in \mathbb{Z}_{p^{*}}^{*}} \operatorname{gcd}\left(p^{k}, a-1\right)^{2}=p^{k-1}\left((p+1) p^{k}-2\right),
$$

and so $g_{1}\left(p^{k}\right)=\left((p+1) p^{k}-2\right) /(p-1)$ as claimed in (2.2). 
(ii) For $p=2$ and $k \geq 2$ we find, using (3.7) in the next-to-last step, that

$$
\begin{aligned}
& g_{2}\left(2^{k}\right) \varphi\left(2^{k}\right)=\sum_{a \in \mathbb{Z}_{2^{*}}^{*}} \operatorname{gcd}\left(2^{k}, a-1\right) \operatorname{gcd}\left(2^{k}, a+1\right) \\
& =\sum_{j=0}^{2^{k-1}-1} \operatorname{gcd}\left(2^{k}, 2 j\right) \operatorname{gcd}\left(2^{k}, 2 j+2\right) \\
& =4 \sum_{j=0}^{2^{k-1}-1} \operatorname{gcd}\left(2^{k-1}, j\right) \operatorname{gcd}\left(2^{k-1}, j+1\right) \\
& =4 \sum_{i=0}^{2^{k-2}-1} \operatorname{gcd}\left(2^{k-1}, 2 i\right) \operatorname{gcd}\left(2^{k-1}, 2 i+1\right) \\
& +4 \sum_{i=0}^{2^{k-2}-1} \operatorname{gcd}\left(2^{k-1}, 2 i+1\right) \operatorname{gcd}\left(2^{k-1}, 2 i+2\right) \\
& =4 \sum_{i=0}^{2^{k-2}-1} \operatorname{gcd}\left(2^{k-1}, 2 i\right)+4 \sum_{i=0}^{2^{k-2}-1} \operatorname{gcd}\left(2^{k-1}, 2 i+2\right) \\
& =8 \sum_{i=0}^{2^{k-2}-1} \operatorname{gcd}\left(2^{k-1}, 2 i\right)=8 \sum_{a \in \mathbb{Z}_{2^{k}-1}^{*}} \operatorname{gcd}\left(2^{k-1}, a-1\right) \\
& =8 k \varphi\left(2^{k-1}\right)=4 k \varphi\left(2^{k}\right),
\end{aligned}
$$

as claimed in (2.3). The case $k=1$ is easily verified directly.

If $p>2$ then at most one of $a-1, a+1$ is divisible by $p$. Hence we find, using (3.7), that

$$
\begin{aligned}
g_{2}\left(p^{k}\right) \varphi\left(p^{k}\right) & =\sum_{a \in \mathbb{Z}_{p^{*}}^{*}} \operatorname{gcd}\left(p^{k}, a-1\right) \operatorname{gcd}\left(p^{k}, a+1\right) \\
& =\sum_{a \in \mathbb{Z}_{p^{*}}^{*}} \operatorname{gcd}\left(p^{k}, a-1\right)+\sum_{a \in \mathbb{Z}_{p^{*}}^{*}} \operatorname{gcd}\left(p^{k}, a+1\right)-\sum_{a \in \mathbb{Z}_{p^{*}}^{*}} 1 \\
& =2(k+1) \varphi\left(p^{k}\right)-\varphi\left(p^{k}\right)=(2 k+1) \varphi\left(p^{k}\right)
\end{aligned}
$$

and (2.3) follows. 
(iii) For $p=2$ and $k \geq 2$ we obtain

$$
\begin{aligned}
g_{3}\left(2^{k}\right) \varphi\left(2^{k}\right) & =\sum_{a \in \mathbb{Z}_{2}^{*}} \operatorname{gcd}\left(2^{k}, a^{2}-1\right)=\sum_{j=0}^{2^{k-1}-1} \operatorname{gcd}\left(2^{k},(2 j+1)^{2}-1\right) \\
& =4 \sum_{j=0}^{2^{k-1}-1} \operatorname{gcd}\left(2^{k-2}, j(j+1)\right) \\
& =4 \sum_{j=0}^{2^{k-1}-1} \operatorname{gcd}\left(2^{k-2}, j\right) \operatorname{gcd}\left(2^{k-2}, j+1\right) \\
& =4 \sum_{j=0}^{2^{k-2}-1} \operatorname{gcd}\left(2^{k-2}, j\right) \operatorname{gcd}\left(2^{k-2}, j+1\right) \\
& +4 \sum_{j=0}^{2^{k-2}-1} \operatorname{gcd}\left(2^{k-2}, j+2^{k-2}\right) \operatorname{gcd}\left(2^{k-2}, j+1+2^{k-2}\right) \\
& =8 \sum_{j=0}^{2^{k-2}-1} \operatorname{gcd}\left(2^{k-2}, j\right) \operatorname{gcd}\left(2^{k-2}, j+1\right) \\
& =8(k-1) \varphi\left(2^{k-1}\right)=4(k-1) \varphi\left(2^{k}\right)
\end{aligned}
$$

by (3.8) and (3.9). The case $k=1$ is easily verified directly.

If $p>2$ then at most one of $a-1, a+1$ is divisible by $p$. It follows that $\operatorname{gcd}\left(p^{k}, a^{2}-\right.$ $1)=\operatorname{gcd}\left(p^{k}, a-1\right) \operatorname{gcd}\left(p^{k}, a+1\right)$, and so $g_{3}\left(p^{k}\right)=g_{2}\left(p^{k}\right)=2 k+1$, proving (2.4).

(iv) For $p=2$ we have

$$
\begin{aligned}
g_{4}\left(2^{k}\right) \varphi\left(2^{k}\right) & =\sum_{a \in \mathbb{Z}_{2^{*}}} \operatorname{gcd}\left(2^{k}, a^{2}+1\right)=\sum_{j=0}^{2^{k-1}-1} \operatorname{gcd}\left(2^{k},(2 j+1)^{2}+1\right) \\
& =2 \sum_{j=0}^{2^{k-1}-1} \operatorname{gcd}\left(2^{k-1}, 2 j^{2}+2 j+1\right)=2 \cdot 2^{k-1}=2 \varphi\left(2^{k}\right) .
\end{aligned}
$$

Assume that $p \equiv 1(\bmod 4)$. Then -1 is a quadratic residue modulo $p^{k}$, so there is an $r \in \mathbb{Z}$ such that $r^{2} \equiv-1\left(\bmod p^{k}\right)$. By Lemma $3.5(\mathrm{i}), \operatorname{gcd}\left(p^{k}, a^{2}+1\right)=\operatorname{gcd}\left(p^{k}, a^{2}-\right.$ $\left.r^{2}\right)$, hence

$$
\begin{aligned}
g_{4}\left(p^{k}\right) \varphi\left(p^{k}\right) & =\sum_{a \in \mathbb{Z}_{p^{*}}^{*}} \operatorname{gcd}\left(p^{k}, a^{2}+1\right)=\sum_{a \in \mathbb{Z}_{p^{*}}^{*}} \operatorname{gcd}\left(p^{k}, a^{2}-r^{2}\right) \\
& =\sum_{a \in \mathbb{Z}_{p^{*}}^{*}} \operatorname{gcd}\left(p^{k},(a-r)(a+r)\right) .
\end{aligned}
$$

If $p \mid a-r$ and $p \mid a+r$ then $p \mid 2 a$ which is false, since $p$ is odd and $a \in \mathbb{Z}_{p^{k}}^{*}$. Hence at most one of $a-r, a+r$ is divisible by $p$. Now by the same argument as in (ii) we find that $g_{4}\left(p^{k}\right) \varphi\left(p^{k}\right)=(2 k+1) \varphi\left(p^{k}\right)$, hence $g_{4}\left(p^{k}\right)=2 k+1$. 
Finally, let $p \equiv 3(\bmod 4)$. Then -1 is a quadratic nonresidue modulo $p$, hence $\operatorname{gcd}\left(p^{k}, a^{2}+1\right)=1$ for all $a$. It follows that

$$
g_{4}\left(p^{k}\right) \varphi\left(p^{k}\right)=\sum_{a \in \mathbb{Z}_{p^{*}}^{*}} \operatorname{gcd}\left(p^{k}, a^{2}+1\right)=\varphi\left(p^{k}\right)
$$

and so $g_{4}\left(p^{k}\right)=1$, proving $(2.5)$.

It remains to show that $g_{1}(n), g_{2}(n), g_{3}(n), g_{4}(n)$ are multiplicative.

Lemma 3.12. Let

$$
g(n)=\sum_{a \in \mathbb{Z}_{n}^{*}} \prod_{k=1}^{r} \operatorname{gcd}\left(n, P_{k}(a)\right)
$$

where $P_{1}(x), P_{2}(x), \ldots, P_{r}(x)$ are polynomials in $x$ with integer coefficients. Then $g(n)$ is a multiplicative arithmetic function.

Proof. Let $n=n_{1} n_{2}$ where $\operatorname{gcd}\left(n_{1}, n_{2}\right)=1$. We need to show that $g(n)=g\left(n_{1}\right) g\left(n_{2}\right)$. For $a \in \mathbb{Z}_{n}$, let $a_{1} \in \mathbb{Z}_{n_{1}}$ and $a_{2} \in \mathbb{Z}_{n_{2}}$ be such that

$$
a \equiv a_{1} \quad\left(\bmod n_{1}\right), \quad a \equiv a_{2} \quad\left(\bmod n_{2}\right) .
$$

By the Chinese Remainder Theorem, the mapping

$$
f: a \mapsto\left(a_{1}, a_{2}\right)
$$

is a bijection from $\mathbb{Z}_{n}$ to $\mathbb{Z}_{n_{1}} \times \mathbb{Z}_{n_{2}}$. By Lemma 3.5 (i) and (ii), $\operatorname{gcd}\left(n_{1} n_{2}, a\right)=1$ iff $\operatorname{gcd}\left(n_{1}, a\right)=\operatorname{gcd}\left(n_{2}, a\right)=1$ iff $\operatorname{gcd}\left(n_{1}, a_{1}\right)=\operatorname{gcd}\left(n_{2}, a_{2}\right)=1$, therefore $f$ restricted to $\mathbb{Z}_{n}^{*}$ is a bijection from $\mathbb{Z}_{n}^{*}$ to $\mathbb{Z}_{n_{1}}^{*} \times \mathbb{Z}_{n_{2}}^{*}$. Also, $P_{k}(a) \equiv P_{k}\left(a_{i}\right)\left(\bmod n_{i}\right)$ for $i=1,2$, hence by Lemma 3.5 (i) and (ii),

$$
\begin{aligned}
\operatorname{gcd}\left(n_{1} n_{2}, P_{k}(a)\right) & =\operatorname{gcd}\left(n_{1}, P_{k}(a)\right) \operatorname{gcd}\left(n_{2}, P_{k}(a)\right) \\
& =\operatorname{gcd}\left(n_{1}, P_{k}\left(a_{1}\right)\right) \operatorname{gcd}\left(n_{2}, P_{k}\left(a_{2}\right)\right) .
\end{aligned}
$$

It follows that

$$
\begin{aligned}
g\left(n_{1} n_{2}\right) & =\sum_{\left(a_{1}, a_{2}\right) \in \mathbb{Z}_{n_{1}}^{*} \times \mathbb{Z}_{n_{2}}^{*}} \prod_{k=1}^{r} \operatorname{gcd}\left(n_{1}, P_{k}\left(a_{1}\right)\right) \operatorname{gcd}\left(n_{2}, P_{k}\left(a_{2}\right)\right) \\
& =\sum_{a_{1} \in \mathbb{Z}_{n_{1}}} \prod_{k=1}^{r} \operatorname{gcd}\left(n_{1}, P_{k}\left(a_{1}\right)\right) \sum_{a_{2} \in \mathbb{Z}_{n_{2}}} \prod_{k=1}^{r} \operatorname{gcd}\left(n_{2}, P_{k}\left(a_{2}\right)\right) \\
& =g\left(n_{1}\right) g\left(n_{2}\right)
\end{aligned}
$$

proving multiplicativity of $g(n)$.

\section{Proof of Theorem 2.1:}

Clearly $I(n)=\left|\mathcal{K}_{n}\right| \simeq \mid$, and the assumptions of Lemma 3.9 are satisfied. We still need to compute $\nu_{0}\left(\mathbb{Z}_{n} \times \mathbb{Z}_{n}\right)$. From Corollary 3.4 (iii) it follows that the set $U_{n}:=$ $\left(\{0\} \times \mathbb{Z}_{n}\right) \cup\left(\mathbb{Z}_{n} \times\{0\}\right)$ equals the union of $\tau(n)$ orbits with representatives $(0, k)$ where $k \mid n$ (with $k=n$ replaced by 0 ). So if $n$ is odd, $\nu_{0}\left(\mathbb{Z}_{n} \times \mathbb{Z}_{n}\right)=\tau(n)$. If $n$ is even, the set $V_{n}:=\left(\{n / 2\} \times \mathbb{Z}_{n}\right) \cup\left(\mathbb{Z}_{n} \times\{n / 2\}\right)$ equals the union of $\tau(n)$ orbits with representatives $(n / 2, k)$ where $k \mid n$ (with $n$ replaced by 0 ). The two sets $U_{n}$ and $V_{n}$ share the orbit containing $(n / 2,0)$, hence in this case $\nu_{0}\left(\mathbb{Z}_{n} \times \mathbb{Z}_{n}\right)=2 \tau(n)-1$. Equation (2.1) now follows by Lemma 3.9, using Proposition 3.10, Lemma 3.11 and Lemma 3.12. 


\subsection{Generalized Petersen graphs}

Let $\mathcal{K}_{n}$ be the set of all generalized Petersen graphs on $2 n$ vertices, and

$$
K_{n}:=\mathbb{Z}_{n}^{*} \times \mathbb{Z}_{n} \cup \mathbb{Z}_{n} \times \mathbb{Z}_{n}^{*}
$$

\section{Proposition 3.13.}

$$
\left|K_{n} / \sim_{\alpha_{n}}\right|=\frac{1}{4}(2 n-\varphi(n)+2 \operatorname{gcd}(n, 2)+r(n)+s(n))
$$

Proof. We use Lemma 3.1. Assume that $(j, k) \in K_{n}$ is fixed by some $g \in G_{n}$.

a) If $g=\xi_{a}$ then $(a j, a k)=(j, k)$. Since $\{j, k\} \cap \mathbb{Z}_{n}^{*} \neq \emptyset$, it follows that $a \equiv 1$ $(\bmod n)$. So $\sum_{a \in \mathbb{Z}_{n}^{*}} \operatorname{fix}_{\alpha_{n}}\left(\xi_{a}\right)=\operatorname{fix}_{\alpha_{n}}\left(\xi_{1}\right)=\left|K_{n}\right|=n^{2}-(n-\varphi(n))^{2}=\varphi(n)(2 n-$ $\varphi(n))$.

b) If $g=\xi_{a} \mu$ then $(a j,-a k)=(j, k)$. Since $\{j, k\} \cap \mathbb{Z}_{n}^{*} \neq \emptyset$, it follows that $a \equiv$ $\pm 1(\bmod n)$. In one case, $2 k \equiv 0(\bmod n)$, so $k=0$ or $k=n / 2$ if $n$ is even, and $j \in \mathbb{Z}_{n}^{*}$. In the other, the roles of $j$ and $k$ are reversed. So $\operatorname{fix}_{\alpha_{n}}\left(\xi_{1} \mu\right)=\operatorname{fix}_{\alpha_{n}}\left(\xi_{-1} \mu\right)=$ $\operatorname{gcd}(n, 2) \varphi(n)$, and $\sum_{a \in \mathbb{Z}_{n}^{*}} \operatorname{fix}_{\alpha_{n}}\left(\xi_{a} \mu\right)=2 \operatorname{gcd}(n, 2) \varphi(n)$.

c) If $g=\xi_{a} \rho$ then $(a k, a j)=(j, k)$. In this case $a^{2} j \equiv j(\bmod n)$ and $a^{2} k \equiv k$ $(\bmod n)$, so $a^{2} \equiv 1(\bmod n), j, k \in \mathbb{Z}_{n}^{*}$, and $k \equiv a j(\bmod n)$ is determined by the choice of $j \in \mathbb{Z}_{n}^{*}$. Thus $\sum_{a \in \mathbb{Z}_{n}^{*}}$ fix $_{\alpha_{n}}\left(\xi_{a} \rho\right)=r(n) \varphi(n)$.

d) If $g=\xi_{a} \rho \mu$ then $(-a k, a j)=(j, k)$. In this case $a^{2} j \equiv-j(\bmod n)$ and $a^{2} k \equiv$ $-k(\bmod n)$, so $a^{2} \equiv-1(\bmod n), j, k \in \mathbb{Z}_{n}^{*}$, and $k \equiv a j(\bmod n)$ is determined by the choice of $j \in \mathbb{Z}_{n}^{*}$. Thus $\sum_{a \in \mathbb{Z}_{n}^{*}} \operatorname{fix}_{\alpha_{n}}\left(\xi_{a} \rho \mu\right)=s(n) \varphi(n)$.

Equation (3.10) now follows from Lemma 3.1.

\section{Proof of Theorem 2.2:}

Clearly $P(n)=\left|\mathcal{K}_{n} / \simeq\right|$. It follows from Theorem 1.1 that $I(n, j, k)$ is isomorphic to a generalized Petersen graph if and only if $j \in \mathbb{Z}_{n}^{*}$ or $k \in \mathbb{Z}_{n}^{*}$, hence the assumptions of Lemma 3.9 are satisfied. We still need to compute $\nu_{0}\left(K_{n}\right)$, the number of orbits containing pairs of the form $(0, k)$ or $(n / 2, k)$ with $k \in \mathbb{Z}_{n}^{*}$. There are two such orbits if $n$ is even, and one if $n$ is odd, hence $\nu_{0}\left(K_{n}\right)=\operatorname{gcd}(n, 2)$. Equation (2.6) now follows by Lemma 3.9, using Proposition 3.13.

\subsection{Connected I-graphs}

Let $\mathcal{K}_{n}$ be the set of all connected I-graphs on $2 n$ vertices, and

$$
K_{n}:=\left\{(j, k) \in \mathbb{Z}_{n} \times \mathbb{Z}_{n} ; \operatorname{gcd}(n, j, k)=1\right\} .
$$

\section{Proposition 3.14.}

$$
\left|K_{n} / \sim_{\alpha_{n}}\right|=\frac{1}{4}\left(\frac{J_{2}(n)}{\varphi(n)}+r(n)+s(n)+t(n)\right)
$$

where $t(n)=t_{1}(n)+t_{2}(n)$ is given in (2.8).

Proof. We use Lemma 3.1. Assume that $(j, k) \in K_{n}$ is fixed by some $g \in G_{n}$.

a) If $g=\xi_{a}$ then $(a j, a k)=(j, k)$. Let $d=\operatorname{gcd}(n, a-1), n=n^{\prime} d$ and $a-1=a^{\prime} d$. As in the proof of Proposition 3.2, we see that $n^{\prime} \mid j$ and $n^{\prime} \mid k$. Since $n^{\prime} \mid n$ as well, it follows 
that $n^{\prime}=1$ and so $n \mid a-1$, which is only possible if $a=1$. Thus $\xi_{a}$ has no fixed points unless $a=1$. As $\xi_{1}$ fixes all points in $K_{n}$, we have

$$
\sum_{a \in \mathbb{Z}_{n}^{*}} \operatorname{fix}_{\alpha_{n}}\left(\xi_{a}\right)=\operatorname{fix}_{\alpha_{n}}\left(\xi_{1}\right)=\left|K_{n}\right|=J_{2}(n) .
$$

b) If $g=\xi_{a} \mu$ then $(a j,-a k)=(j, k)$. Denote $n_{j}=\operatorname{gcd}(n, j)$ and $n_{k}=\operatorname{gcd}(n, k)$. Any common divisor of $n_{j}$ and $n_{k}$ is a common divisor of $n, j, k$, hence $n_{j} \perp n_{k}$ and $n_{j} n_{k} \mid n$. Denote $n_{0}=n /\left(n_{j} n_{k}\right), j^{\prime}=j / n_{j}, k^{\prime}=k / n_{k}$. Then

$$
n=n_{0} n_{j} n_{k}, j^{\prime} \in \mathbb{Z}_{n_{0} n_{k}}^{*}, k^{\prime} \in \mathbb{Z}_{n_{0} n_{j}}^{*} .
$$

From $a j \equiv j(\bmod n)$ it follows that $n_{0} n_{k} \mid(a-1) j^{\prime}$, hence $n_{0} n_{k} \mid a-1$. From $a k \equiv-k$ $(\bmod n)$ it follows that $n_{0} n_{j} \mid(a+1) k^{\prime}$, hence $n_{0} n_{j} \mid a+1$. Therefore $n_{0} \mid 2$, and so $n_{0} \in\{1,2\}$ and $\varphi\left(n_{0}\right)=1$.

We claim that for each pair $(j, k)$ where $j=j^{\prime} n_{j}, k=k^{\prime} n_{k}, n=n_{0} n_{j} n_{k}, n_{0} \in\{1,2\}$, $n_{j} \perp n_{k}, j^{\prime} \in \mathbb{Z}_{n_{0} n_{k}}^{*}$ and $k^{\prime} \in \mathbb{Z}_{n_{0} n_{j}}^{*}$, there is a unique $a \in \mathbb{Z}_{n}^{*}$ such that $a j \equiv j(\bmod n)$ and $a k \equiv-k(\bmod n)$. Indeed, let $n=\prod_{i=1}^{m} p_{i}^{e_{i}}$ be the prime factorization of $n$ (i.e., $p_{1}, p_{2}, \ldots, p_{m}$ are distinct primes and $e_{i} \geq 1$ for $\left.i=1,2, \ldots, m\right)$. Define $a \in \mathbb{Z}$ by requiring that for each $i \in\{1,2, \ldots, m\}$,

$$
\begin{array}{ll}
a \equiv-1\left(\bmod p_{i}^{e_{i}}\right) & \text { if } p_{i}^{e_{i}} \mid n_{0} n_{j}, \\
a \equiv 1\left(\bmod p_{i}^{e_{i}}\right) & \text { if } p_{i}^{e_{i}} \mid n_{0} n_{k} .
\end{array}
$$

At least one of $p_{i}^{e_{i}} \mid n_{0} n_{j}$ and $p_{i}^{e_{i}} \mid n_{0} n_{k}$ holds for each $i \in\{1,2, \ldots, m\}$, and both hold only if $p_{i}^{e_{i}}=n_{0}=2$, hence these requirements are consistent, and by the Chinese Remainder Theorem, there is a unique $a \in \mathbb{Z}_{n}$ which satisfies them. In fact, $a^{2} \equiv 1\left(\bmod p_{i}^{e_{i}}\right)$ for $i=1,2, \ldots, m$, hence $a^{2} \equiv 1(\bmod n)$, and so $a \in \mathbb{Z}_{n}^{*}$. Note that $a$ is odd if $n_{0}=2$, therefore $n_{0} \mid a-1$ and $n_{0} \mid a+1$.

If $p_{i}^{e_{i}} \mid n_{0} n_{j}$ then $p_{i}^{e_{i}}\left|n_{0} j\right|(a-1) j$. Also, $a \equiv-1\left(\bmod p_{i}^{e_{i}}\right)$, so $p_{i}^{e_{i}} \mid(a+1) k$.

If $p_{i}^{e_{i}} \mid n_{0} n_{k}$ then $p_{i}^{e_{i}}\left|n_{0} k\right|(a+1) k$. Also, $a \equiv 1\left(\bmod p_{i}^{e_{i}}\right)$, so $p_{i}^{e_{i}} \mid(a-1) j$.

In either case, $p_{i}^{e_{i}} \mid(a-1) j$ and $p_{i}^{e_{i}} \mid(a+1) k$. As this holds for all $i \in\{1,2, \ldots, m\}$, it follows that $n \mid(a-1) j$ and $n \mid(a+1) k$, hence $a j \equiv j(\bmod n)$ and $a k \equiv-k(\bmod n)$ as claimed.

Thus to construct $(j, k) \in K_{n}$ which is fixed by some $\xi_{a} \mu$, first select $n_{0}, n_{j}, n_{k}, j^{\prime}$, $k^{\prime} \in \mathbb{Z}_{n}$ such that $n_{0} \in\{1,2\}, n_{j} \perp n_{k}, n=n_{0} n_{j} n_{k}, j^{\prime} \in \mathbb{Z}_{n_{0} n_{k}}^{*}$ and $k^{\prime} \in \mathbb{Z}_{n_{0} n_{j}}^{*}$, then take $j=j^{\prime} n_{j}, k=k^{\prime} n_{k}$. This can be done in

$$
\sum_{n_{0} \in\{1,2\}, n_{j} \perp n_{k}, n=n_{0} n_{j} n_{k}} \varphi\left(n_{0} n_{k}\right) \varphi\left(n_{0} n_{j}\right)
$$

ways. W.l.g. assume that $n_{k}$ is odd. Then $\varphi\left(n_{0} n_{k}\right) \varphi\left(n_{0} n_{j}\right)=\varphi\left(n_{0}\right) \varphi\left(n_{k}\right) \varphi\left(n_{0} n_{j}\right)=$ $\varphi\left(n_{k}\right) \varphi\left(n_{0} n_{j}\right)=\varphi\left(n_{0} n_{j} n_{k}\right)=\varphi(n)$, hence

$$
\sum_{a \in \mathbb{Z}_{n}^{*}} \operatorname{fix}_{\alpha_{n}}\left(\xi_{a} \mu\right)=\varphi(n)\left(t_{1}(n)+t_{2}(n)\right)
$$

where $t_{n_{0}}(n)=\left|\left\{\left(n_{j}, n_{k}\right) ; n_{j} \perp n_{k}, n=n_{0} n_{j} n_{k}\right\}\right|$. Clearly, $t_{1}(n)=2^{\omega(n)}$ and

$$
t_{2}(n)= \begin{cases}2^{\omega(n / 2)}, & n \text { even } \\ 0, & n \text { odd }\end{cases}
$$


c) If $g=\xi_{a} \rho$ then $(a k, a j)=(j, k)$. In this case $\operatorname{gcd}(n, j, a j)=\operatorname{gcd}(n, j, k)=1$ by Lemma $3.5(\mathrm{i})$, and $a^{2} j \equiv j(\bmod n)$. It follows that $j \in \mathbb{Z}_{n}^{*}$ and $a^{2} \equiv 1(\bmod n)$. Since $k \equiv a j(\bmod n)$ is determined by the choice of $j \in \mathbb{Z}_{n}^{*}$, we have $\sum_{a \in \mathbb{Z}_{n}^{*}} \operatorname{fix}_{\alpha_{n}}\left(\xi_{a} \rho\right)=$ $r(n) \varphi(n)$.

d) If $g=\xi_{a} \rho \mu$ then $(-a k, a j)=(j, k)$. In this case $\operatorname{gcd}(n, j, a j)=\operatorname{gcd}(n, j, k)$ $=1$ by Lemma $3.5(\mathrm{i})$, and $a^{2} j \equiv-j(\bmod n)$. It follows that $j \in \mathbb{Z}_{n}^{*}$ and $a^{2} \equiv-1$ $(\bmod n)$. Since $k \equiv a j(\bmod n)$ is determined by the choice of $j \in \mathbb{Z}_{n}^{*}$, we have $\sum_{a \in \mathbb{Z}_{n}^{*}} \operatorname{fix}_{\alpha_{n}}\left(\xi_{a} \rho \mu\right)=s(n) \varphi(n)$.

Equation (3.11) now follows from Lemma 3.1.

\section{Proof of Theorem 2.3:}

Clearly $I_{c}(n)=\left|\mathcal{K}_{n} / \simeq\right|$. It follows from Theorem 1.2 that the assumptions of Lemma 3.9 are satisfied. We still need to compute $\nu_{0}\left(K_{n}\right)$, the number of orbits containing pairs of the form $(0, k)$ or $(n / 2, k)$ with $k \in \mathbb{Z}_{n}^{*}$.

If $(0, k) \in K_{n}$ then $\operatorname{gcd}(n, k)=\operatorname{gcd}(n, 0, k)=1$, hence $k \in \mathbb{Z}_{n}^{*}$. It follows that all such pairs belong to a single orbit of $\alpha_{n}$.

Assume that $n \equiv 0(\bmod 4)$. If $(n / 2, k) \in K_{n}$ then $\operatorname{gcd}(n, n / 2, k)=1$. Since in this case $\operatorname{gcd}(n, n / 2, k)=1$ iff $\operatorname{gcd}(n, k)=1$, it follows that $k \in \mathbb{Z}_{n}^{*}$. For any $a \in \mathbb{Z}_{n}^{*}$ we have $a(n / 2) \equiv n / 2(\bmod n)$, hence we conclude again that all such pairs belong to a single orbit of $\alpha_{n}$.

Assume that $n \equiv 2(\bmod 4)$. If $(n / 2, k) \in K_{n}$ then $\operatorname{gcd}(n, n / 2, k)=1$. In this case it is straightforward to see that $\operatorname{gcd}(n, n / 2, k)=1$ iff $k=2^{j} a$ for some $j \geq 0$ and $a \in \mathbb{Z}_{n}^{*}$. All the pairs $(n / 2, a)$ with $a \in \mathbb{Z}_{n}^{*}$ clearly belong to a single orbit of $\alpha_{n}$. Now we claim that $4 \mathbb{Z}_{n}^{*}=2 \mathbb{Z}_{n}^{*}$. Indeed, let $q=n / 2$ and $a \in \mathbb{Z}_{n}^{*}$. Then $\operatorname{gcd}(2 a+q, n)=1$ and $4 a \equiv 2(2 a+q)(\bmod n)$, proving that $4 \mathbb{Z}_{n}^{*} \subseteq 2 \mathbb{Z}_{n}^{*}$. Conversely, if $q \equiv 1(\bmod 4)$ then $\operatorname{gcd}((q+1) / 2, n)=1$ and $2 a \equiv 4 a(q+1) / 2(\bmod n)$. If $q \equiv 3(\bmod 4)$ then $\operatorname{gcd}((3 q+1) / 2, n)=1$ and $2 a \equiv 4 a((3 q+1) / 2)(\bmod n)$, proving that $2 \mathbb{Z}_{n}^{*} \subseteq 4 \mathbb{Z}_{n}^{*}$, and also the claim. Hence all the pairs $\left(n / 2,2^{j} a\right)$ with $j \geq 1$ and $a \in \mathbb{Z}_{n}^{*}$ also belong to a single orbit of $\alpha_{n}$. On the other hand, all the pairs in the orbit of $(n / 2,1)$ have one component in $\mathbb{Z}_{n}^{*}$, while all the pairs in the orbit of $(n / 2,2)$ have neither component in $\mathbb{Z}_{n}^{*}$, hence these two orbits are distinct.

It follows that

$$
\nu_{0}\left(K_{n}\right)= \begin{cases}1, & n \equiv 1 \quad(\bmod 2) \\ 2, & n \equiv 0 \quad(\bmod 4) \\ 3, & n \equiv 2 \quad(\bmod 4)\end{cases}
$$

which together with Lemma 3.9 and Proposition 3.14 yields (2.7).

\subsection{Bipartite generalized Petersen graphs}

Let $\mathcal{K}_{n}$ be the set of all bipartite generalized Petersen graphs on $2 n$ vertices, and

$$
K_{n}:=\mathbb{Z}_{n}^{*} \times \mathbb{Z}_{n}^{o} \cup \mathbb{Z}_{n}^{o} \times \mathbb{Z}_{n}^{*},
$$

where $\mathbb{Z}_{n}^{o}$ is the subset of odd elements in $\mathbb{Z}_{n}$.

Proposition 3.15. Let $n$ be even. Then

$$
\left|K_{n} / \sim_{\alpha_{n}}\right|=\frac{1}{4}(n-\varphi(n)+2((n / 2) \bmod 2)+r(n)+s(n)) .
$$


Proof. We follow the proof of Proposition 3.13. Assume that $(j, k) \in K_{n}$ is fixed by some $g \in G_{n}$, and notice that both $j$ and $k$ are odd.

a) If $g=\xi_{a}$ then $(a j, a k)=(j, k)$. From $\{j, k\} \cap \mathbb{Z}_{n}^{*} \neq \emptyset$ it follows that $a \equiv 1(\bmod n)$. So $\sum_{a \in \mathbb{Z}_{n}^{*}} \operatorname{fix}_{\alpha_{n}}\left(\xi_{a}\right)=\left|K_{n}\right|=(n / 2)^{2}-(n / 2-\varphi(n))^{2}=\varphi(n)(n-\varphi(n))$.

b) If $g=\xi_{a} \mu$ then $(a j,-a k)=(j, k)$. If $j \in \mathbb{Z}_{n}^{*}$, then $a \equiv 1(\bmod n)$ and $2 k \equiv$ $0(\bmod n)$. As $k$ is odd, this is only possible if $n \neq 0(\bmod 4)$ and $k=n / 2$. If $k \in \mathbb{Z}_{n}^{*}$, then $a \equiv-1(\bmod n), n \not \equiv 0(\bmod 4)$ and $j=n / 2$. So $\operatorname{fix}_{\alpha_{n}}\left(\xi_{1} \mu\right)=\operatorname{fix}_{\alpha_{n}}\left(\xi_{-1} \mu\right)=$ $\varphi(n)(n / 2(\bmod 2))$, and $\sum_{a \in \mathbb{Z}_{n}^{*}} \operatorname{fix}_{\alpha_{n}}\left(\xi_{a} \mu\right)=2 \varphi(n)(n / 2(\bmod 2))$.

c), d): As in the proof of Proposition 3.13.

Equation (3.12) now follows from Lemma 3.1.

Proof of Theorem 2.4:

Clearly $P_{b}(n)=\left|\mathcal{K}_{n}\right| \simeq \mid$. It follows from Theorems 1.1 and 1.3 that $I(n, j, k)$ is isomorphic to a bipartite generalized Petersen graph if and only if $j \in \mathbb{Z}_{n}^{*}$ and $k$ is odd, or $k \in \mathbb{Z}_{n}^{*}$ and $j$ is odd, hence the assumptions of Lemma 3.9 are satisfied. We still need to compute $\nu_{0}\left(K_{n}\right)$, the number of orbits containing pairs of the form $(n / 2, k)$ with $n / 2$ odd and $k \in \mathbb{Z}_{n}^{*}$. There are no such orbits if $n \equiv 0(\bmod 4)$, and one such orbit if $n \equiv 2$ $(\bmod 4)$. Hence

$$
\nu_{0}\left(K_{n}\right)=(n / 2) \bmod 2,
$$

which together with Lemma 3.9 and Proposition 3.15 yields (2.9).

\subsection{Bipartite connected I-graphs}

Let $\mathcal{K}_{n}$ be the set of all bipartite connected I-graphs on $2 n$ vertices, and

$$
K_{n}:=\left\{(j, k) \in \mathbb{Z}_{n} \times \mathbb{Z}_{n} ; \operatorname{gcd}(n, j, k)=1, j, k \text { odd }\right\} .
$$

Proposition 3.16. Let $n$ be even. Then

$$
\left|K_{n} / \sim_{\alpha_{n}}\right|=\frac{1}{4}\left(\frac{J_{2}(n)}{3 \varphi(n)}+((n / 2) \bmod 2) 2^{\omega(n / 2)}+r(n)+s(n)\right) .
$$

Proof. We follow the proof of Proposition 3.14. Assume that $(j, k) \in K_{n}$ is fixed by some $g \in G_{n}$.

a) If $g=\xi_{a}$ then $(a j, a k)=(j, k)$. As in case a) in the proof of Proposition 3.14, we see that $a \equiv 1(\bmod n)$, thus $\sum_{a \in \mathbb{Z}_{n}^{*}} \operatorname{fix}_{\alpha_{n}}\left(\xi_{a}\right)=\operatorname{fix}_{\alpha_{n}}\left(\xi_{1}\right)=\left|K_{n}\right|$. Let

$$
\begin{aligned}
U_{n} & :=\left\{(j, k) \in \mathbb{Z}_{n} \times \mathbb{Z}_{n} ; \operatorname{gcd}(n, j, k)=1, j \text { odd, } k \text { even }\right\}, \\
V_{n} & :=\left\{(j, k) \in \mathbb{Z}_{n} \times \mathbb{Z}_{n} ; \operatorname{gcd}(n, j, k)=1, j \text { even, } k \text { odd }\right\}, \\
W_{n} & :=\left\{(j, k) \in \mathbb{Z}_{n} \times \mathbb{Z}_{n} ; \operatorname{gcd}(n, j, k)=1\right\} .
\end{aligned}
$$

Define the functions $f_{n}: K_{n} \rightarrow U_{n}$ and $g_{n}: U_{n} \rightarrow K_{n}$ by

$$
\begin{aligned}
& f_{n}(j, k):=(j, k+j)(\bmod n), \\
& g_{n}(j, k):=(j, k-j)(\bmod n) .
\end{aligned}
$$

Clearly $\operatorname{gcd}(n, j, k)=1$ iff $\operatorname{gcd}(n, j, k+j)=1$ iff $\operatorname{gcd}(n, j, k-j)=1$. Next, for $j, k$ odd, $k+j(\bmod n)$ is even, and if $j$ is odd and $k$ is even, then $k-j(\bmod n)$ is odd. 
Since $f_{n}\left(g_{n}(j, k)\right)=(j, k)=g_{n}\left(f_{n}(j, k)\right)$, we conclude that $f_{n}$ and $g_{n}$ are bijections, and $\left|K_{n}\right|=\left|U_{n}\right|$. Since $W_{n}=K_{n} \cup U_{n} \cup V_{n},\left|W_{n}\right|=J_{2}(n)$, and $\left|U_{n}\right|=\left|V_{n}\right|$ by symmetry, it follows that $\left|K_{n}\right|=\left|U_{n}\right|=\left|V_{n}\right|=J_{2}(n) / 3$.

b) If $g=\xi_{a} \mu$ then $(a j,-a k)=(j, k)$. As in case b) in the proof of Proposition 3.14, we see that $n=n_{0} n_{j} n_{k}$ where $n_{0}\left|2, n_{j}\right| j$ and $n_{k} \mid k$. Since $n$ is even while $j$ and $k$ are odd, it follows that $n_{0}=2$, hence $\xi_{a} \mu$ has no fixed points if $n \equiv 0(\bmod 4)$. So assume that $n \equiv 2(\bmod 4)$. To construct $(j, k) \in K_{n}$ which is fixed by some (uniquely determined) $\xi_{a} \mu$, first select $n_{j}, n_{k}, j^{\prime}, k^{\prime} \in \mathbb{Z}_{n}$ such that $n_{j} \perp n_{k}, n=2 n_{j} n_{k}, j^{\prime} \in \mathbb{Z}_{2 n_{k}}^{*}$ and $k^{\prime} \in \mathbb{Z}_{2 n_{j}}^{*}$, then take $j=j^{\prime} n_{j}, k=k^{\prime} n_{k}$. This can be done in

$$
\sum_{n_{j} \perp n_{k}, n=2 n_{j} n_{k}} \varphi\left(2 n_{k}\right) \varphi\left(2 n_{j}\right)
$$

ways. Since $n_{k}$ and $n_{j}$ are odd, $\varphi\left(2 n_{k}\right) \varphi\left(2 n_{j}\right)=\varphi\left(n_{k}\right) \varphi\left(2 n_{j}\right)=\varphi\left(2 n_{k} n_{j}\right)=\varphi(n)$. Therefore $\sum_{a \in \mathbb{Z}_{n}^{*}} \operatorname{fix}_{\alpha_{n}}\left(\xi_{a} \mu\right)=\varphi(n) 2^{\omega(n / 2)}$ if $n \equiv 2(\bmod 4)$. By multiplying this expression with $(n / 2)$ mod 2 we extend its validity to all even $n$.

c), d): As in the proof of Proposition 3.14.

Equation (3.13) now follows from Lemma 3.1.

\section{Proof of Theorem 2.5:}

Clearly $I_{b c}(n)=\left|\mathcal{K}_{n} / \simeq\right|$. It follows from Theorems 1.2 and 1.3 that the assumptions of Lemma 3.9 are satisfied. We still need to compute $\nu_{0}\left(K_{n}\right)$, the number of orbits containing pairs of the form $(n / 2, k)$ with $n / 2$ and $k$ odd and $\operatorname{gcd}(n, n / 2, k)=1$. In this case $\operatorname{gcd}(n, n / 2, k)=1$ if and only if $\operatorname{gcd}(n, k)=1$. Therefore there are no such orbits if $n \equiv 0(\bmod 4)$, and one such orbit if $n \equiv 2(\bmod 4)$. Hence

$$
\nu_{0}\left(K_{n}\right)=(n / 2) \bmod 2,
$$

which together with Lemma 3.9 and Proposition 3.16 yields (2.10).

\section{Concluding remark}

It is not difficult to see that the numbers $I_{c}(n)$ and $I(n)$ of isomorphism classes of connected I-graphs resp. all I-graphs on $2 n$ vertices satisfy the pair of Moebius inverse relations

$$
I(n)=\sum_{d \mid n} I_{c}(d), \quad I_{c}(n)=\sum_{d \mid n} \mu(n / d) I(d)
$$

(cf. [8, Sec. 3]).

\section{Acknowledgement}

The authors wish to express their thanks to the referees for their careful reading of the paper and useful suggestions.

\section{References}

[1] M. Boben, T. Pisanski and A. Žitnik, I-graphs and the corresponding configurations, J. Combin. Des. 13 (2005), 406-424. 
[2] I. Z. Bouwer, W. W. Chernoff, B. Monson and Z. Star, The Foster Census, Charles Babbage Research Centre, 1988.

[3] R. Frucht, J. E. Graver and M. E. Watkins, The groups of the generalized Petersen graphs, Proc. Camb. Phil. Soc. 70 (1971), 211-218.

[4] B. Horvat, T. Pisanski and A. Žitnik, All generalized Petersen graphs are unit-distance graphs, submitted.

[5] B. Horvat, T. Pisanski and A. Žitnik, Isomorphism checking of I-graphs, submitted.

[6] V. A. Liskovets and A. D. Mednykh, On the number of connected and disconnected coverings over a manifold, Ars Math. Contemp. 2 (2009), 181-189.

[7] A. Orbanić, M. Petkovšek, T. Pisanski and P. Potočnik, A note on enumeration of one-vertex maps, Ars Math. Contemp., to appear.

[8] M. Petkovšek and T. Pisanski, Counting disconnected structures: chemical trees, fullerenes, I-graphs, and others, Croat. Chem. Acta 78 (2005), 563-567.

[9] T. Pisanski, D. Schattschneider and B. Servatius, Applying Burnside's lemma to a onedimensional Escher problem, Math. Mag. 79 (2006), 167-180.

[10] N. J. A. Sloane, The On-Line Encyclopedia of Integer Sequences, published electronically at http://www.research.att.com/ njas/sequences/.

[11] R. P. Stanley, Enumerative Combinatorics, vol. 2, Cambridge University Press, Cambridge, 1999.

[12] A. Steimle and W. Staton, The isomorphism classes of the generalized Petersen graphs, Discrete Math. 309 (2009), 231-237.

[13] M. E. Watkins, A theorem on Tait colorings with an application to the generalized Petersen graphs, J. Comb. Theory 6 (1969), 152-164. 